\title{
Energy Output Problem
}

National Cancer Institute

\section{Source}

National Cancer Institute. Energy Output Problem. NCI Thesaurus. Code C63002.

Problem with the device's intended output of energy. 